\title{
Die staatliche Finanzierung von Religionsgemeinschaften
}

\section{Lorenz Engi *}

In vielen Kantonen erhalten öffentlich-rechtlich anerkannte Religionsgemeinschaften staatliche Beiträge. Diese Unterstützung wird heute überwiegend mit Leistungen dieser Gemeinschaften begründet, die im gesamtgesellschaftlichen Interesse liegen. Auch nicht-anerkannte Gemeinschaften erbringen möglicherweise solche Leistungen. Daher werden in der Literatur und in der Praxis Möglichkeiten der Weiterentwicklung des Finanzierungssystems diskutiert.

I. Einführung 272

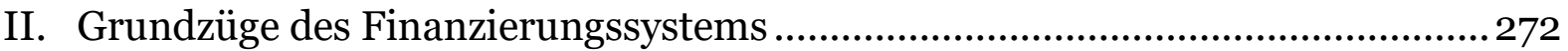

1. Rechtlicher Rahmen ....................................................................... 272

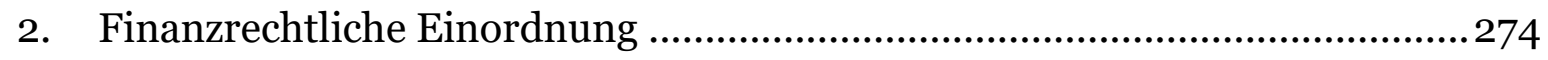

3. Bezug zum Anerkennungssystem ....................................................... 275

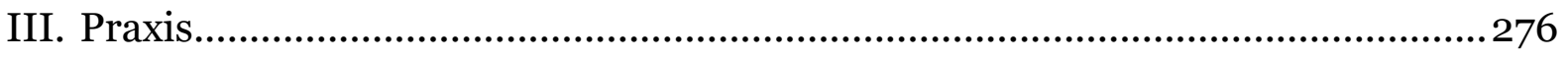

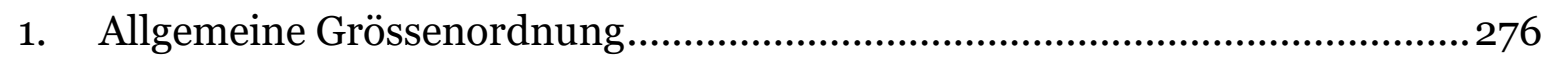

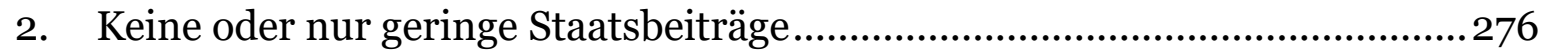

3. Hohe Beiträge in drei Kantonen ............................................................ 277

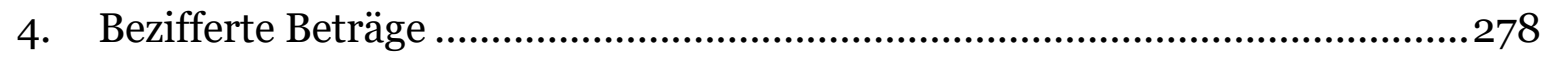

5. Autonomie der Gemeinden ............................................................278

6. Weitere Systeme, insbesondere Unterstützung bestimmter Leistungen .......279

IV. Problematik............................................................................................... 280

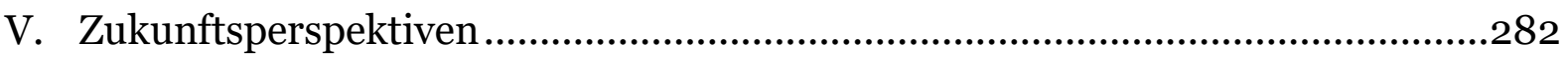

Zitiervorschlag: Lorenz Engi, Die staatliche Finanzierung von Religionsgemeinschaften, in: sui-generis 2018, S. 271

URL: $\quad$ sui-generis.ch/73

DOI: $\quad$ https://doi.org/10.21257/sg.73

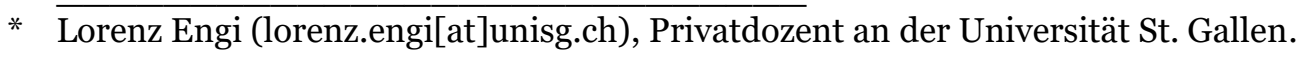




\section{Einführung}

1 Geld ist (hoffentlich) nicht das Erste, an das man denkt, wenn es um die Verhältnisse zwischen Staat und Religionsgemeinschaften geht. Doch wie in allen anderen Bereichen des Lebens, sind die Finanzen auch in diesen Belangen ein wichtiger Faktor. In der Regel stehen dabei die Kirchensteuern im Mittelpunkt. Die öffentlich-rechtlich anerkannten Religionsgemeinschaften dürfen Steuern erheben. In zahlreichen Kantonen werden neben natürlichen auch juristische Personen besteuert ${ }^{1}$. Besonders weil letztere keinen religiösen Glauben haben, wird diese Praxis in der juristischen Literatur sehr verbreitet kritisiert ${ }^{2}$.

Während das Steuerprivileg der Kirchen allgemein bekannt ist und gerade im $\mathrm{Zu}$ sammenhang mit der Besteuerung juristischer Personen oft diskutiert wird3, findet ein anderer Aspekt der finanziellen Beziehungen zwischen Staat und Religionsgemeinschaften weit weniger Beachtung: der Umstand, dass anerkannte Kirchen und Religionsgemeinschaften direkte finanzielle Unterstützung des Staates erhalten. Neben den Kirchensteuern und unabhängig von diesen unterstützen zahlreiche Kantone die anerkannten Re-

\footnotetext{
Umfassende aktuelle Darstellung dazu bei Raimund Süess/Christian R. Tappenbeck/René Pahud de Mortanges, Die Kirchensteuern juristischer Personen in der Schweiz. Eine Dokumentation, Zürich/Basel/Genf 2013.

2 Nachweise bei Lorenz Engi, Die religiöse und ethische Neutralität des Staates. Theoretischer Hintergrund, dogmatischer Gehalt und praktische Bedeutung eines Grundsatzes des schweizerischen Staatsrechts, Habil., Zürich 2017, S. 246 f. (dort Fn. 181).

3 In mehreren Kantonen fanden in den letzten Jahren Abstimmungen dazu statt: René Pahud de Mortanges, in: Bernhard Waldmann/Eva Maria Belser/Astrid Epiney (Hrsg.), Bundesverfassung - Basler Kommentar, Basel 2015, Art. 15 Rz. 56.
}

ligionsgemeinschaften mit Staatsbeiträgen. Die folgenden Ausführungen möchten diesen Aspekt der Beziehungen zwischen Staat und Religionsgemeinschaften beleuchten.

3 Zunächst soll die bestehende Praxis dargestellt werden. Dazu wird zunächst das System in seinen Grundzügen beschrieben (nachfolgend Kap. II), daraufhin sollen die Verhältnisse in den einzelnen Kantonen näher betrachtet werden (Kap. III). Dieser Bestandesaufnahme schliesst sich eine Analyse an. Sie wird zunächst einige Probleme benennen (Kap. IV), um danach Möglichkeiten der Weiterentwicklung zu erörtern (Kap. V).

\section{Grundzüge des Finanzierungssystems}

\section{Rechtlicher Rahmen}

4 Die Mitfinanzierung von anerkannten Religionsgemeinschaften aus allgemeinen Steuermitteln ist in der Schweiz weit verbreitet. Da die allgemeinen Steuermittel auch von Personen stammen, die den betreffenden Religionsgemeinschaften nicht angehören, unterliegt die Förderung dieser Gemeinschaften gewissen Beschränkungen. Insbesondere dürfen allgemeine Steuermittel nicht für eigentliche Kultuszwecke von Religionsgemeinschaften verwendet werden4. Der Begriff

\footnotetext{
4 BGE 107 Ia 126 E. 2b und 3a S. 130 f. Die BV von 1874 hatte die Vorschrift enthalten (Art. 49 Abs. 6 $\mathrm{aBV})$ : «Niemand ist gehalten, Steuern zu zahlen, welche speziell für eigentliche Kultuszwecke einer Religionsgemeinschaft, der er nicht angehört, auferlegt werden.» Dazu Peter Karlen, Das Grundrecht der Religionsfreiheit in der Schweiz, Diss. Zürich 1988, S. 348 ff. Obgleich in der neuen BV nicht mehr enthalten, gilt der Grundsatz weiterhin: Giovanni Biaggini, Bundesverfassung der Schweizerischen Eidgenossenschaft - Kommentar, 2. Aufl., Zürich 2017, Art. 15 Rz. 11.
} 
der Kultuszwecke bzw. kultischen Zwecke ist nicht sehr präzis5. In einem allerdings schon älteren Entscheid fasste das Bundesgericht darunter beispielsweise den Bau und Unterhalt von Kirchen und Pfarrhäusern sowie die Besoldung von Geistlichen und Kirchendienern 6 .

5 Das Bundesgericht lässt es zu, dass kantonale Steuererträge auch für die Bezahlung von Pfarrerlöhnen verwendet werden7. Die Einschränkung, dass allgemeine Steuermittel nicht für Kultuszwecke von Religionsgemeinschaften eingesetzt werden dürfen, gilt somit nur auf Gemeindeebene ${ }^{8}$. Das führt beispielsweise dazu, dass im Kanton Bern die Löhne von Pfarrerinnen und Pfarrern aus kantonalen Steuermitteln bezahlt werden. Die Beschwerde einer Frau, die sich gegen diese Praxis zur Wehr setzte, wies das Bundesgericht 2011 ab. Die Beschwerdeführerin war aus der evangelisch-reformierten Landeskirche ausgetreten und beantragte eine Reduktion ihres Kantonssteueranteils um o,813\% den Anteil der Pfarrerlöhne am Gesamtaufwand des Kantons. Das Bundesgericht führte dazu unter anderem ins Feld, dass nur ein verschwindend kleiner Anteil des kantonalen Budgets für Kultuszwecke verwendet würde9. Die Pflicht zur Bezahlung der Kantonssteuer greife daher nicht in die Glaubens- und Gewissens-

5 Tobias Jaag, Neuordnung des Verhältnisses zwischen Kirchen und Staat im Kanton Zürich, in: Schweizerische Juristen-Zeitung 2003, S. 549558,555 .

6 BGE 107 Ia 126 E. 3 b S. 131.

7 BGE 107 Ia 126 E. 2b S. 130.

8 Felix Hafner/Kathrin Ebnöther, Staatliche Förderung religiöser Aktivitäten, in: René Pahud de Mortanges/Erwin Tanner (Hrsg.), Kooperation zwischen Staat und Religionsgemeinschaften nach staatlichem Recht, Zürich 2005, S. 131-161, 148; Karlen (Fn. 4), S. 351.

$9 \quad$ BGE 138 I 55 E. 3.1 S. 58. freiheit der Beschwerdeführerin ein ${ }^{10}$. Selbst wenn von einem Eingriff in die Religionsfreiheit ausgegangen würde, so das Bundesgericht weiter, wäre dieser unter den Voraussetzungen von Art. 36 BV zulässigi1.

6 Ihren historischen Ursprung haben die staatlichen Leistungen an die kirchlichen Körperschaften in vielen Kantonen darin, dass in früherer Zeit Kirchengut säkularisiert, also in staatlichen Besitz überführt wurde. Daraus ergaben sich Rechtsansprüche der Kirchen, sogenannte historische Rechtstitel. Meist betraf das historisch nur eine Kirche, aus Paritätsgründen wurde die Finanzierung aber später auf die konfessionelle Minderheit ausgedehnt ${ }^{12}$. In den reformierten Gebieten hatte der Staat von der Reformationszeit an Patronate übernommen; er wurde zum wichtigsten Patronatsherrn ${ }^{13}$. Mit den Patronaten ging die Pflicht einher, den Geistlichen die Mittel für einen angemessenen Lebensunterhalt zu gewähren. Im 19. Jahrhundert wurden die Pfründen verkauft und ein neues System staatlicher Pfarrerbesoldung geschaffen ${ }^{14}$. Auf dieser historischen Grundlage haben manche Kantone bis in die jüngste Zeit die Löhne der Pfarrerinnen und Pfarrer bezahlt; vereinzelt ist das bis heu-

10 BGE 138 I 55 E. 3.3 S. 59.

11 BGE 138 I 55 E. 3.4 S. 60.

12 Urs Josef Cavelti/Andreas Kley, Kommentar zu Art. 15, in: Bernhard Ehrenzeller/Benjamin Schindler/Rainer J. Schweizer/Klaus A. Vallender (Hrsg.), St. Galler Kommentar zur Bundesverfassung, 3. Aufl., St. Gallen/Zürich 2014, Rz. 37.

13 Vgl. Christina Schmid-Tschirren, Von der Säkularisation zur Separation. Der Umgang des Staates mit den Kirchengütern in den evangelischreformierten und paritätischen Kantonen der Schweiz im 19. Jahrhundert, Habil. Bern, Zürich/Basel/Genf 2011, S. $58 \mathrm{ff}$.

14 Schmid-Tschirren (Fn. 13), S. 158 ff. 
te der Fall. Im Rahmen neuerer Entflechtungen wurden Leistungen in dieser Form aber überwiegend eingestellt und ein Ausgleich in Form von Staatsbeiträgen geschaffen. Diese werden heute überwiegend mit den Leistungen der Kirchen von gesamtgesellschaftlicher Bedeutung begründet ${ }^{15}$.

7 Bei der Festlegung der Beiträge bleiben die historischen Hintergründe jedoch vielfach prägend, wie beispielsweise die Regelung im Kanton Zürich zeigt: Dieser nahm mit der Kantonsverfassung von $2005^{16}$ und dem Kirchengesetz von 2007 eine Umstellung auf ein System der Kostenbeiträge vor, das an die gesellschaftlich wichtigen Leistungen der Kirchen anknüpft. Eine Übergangsbestimmung der Zürcher Verfassung (Art. 145 Abs. 1 KV ZH) schreibt jedoch vor: «Die auf historischen Rechtstiteln beruhenden Leistungen des Staates an die kirchlichen Körperschaften bleiben bis zur gesetzlichen Neuregelung garantiert. Die Neuregelung dieser Leistungen orientiert sich an deren bisherigem Gesamtumfang.» Die früheren Leistungen aufgrund historischer Rechtstitel waren somit auch für die Höhe der Unterstützung im neuen System massgebend ${ }^{17}$.

15 Vgl. z.B. $§ 19$ Abs. 1 des Kirchengesetzes des Kantons Zürich vom 9. Juli 2007 (KiG; LS 180.1): «Der Kanton bewilligt mit einem Globalbudget Kostenbeiträge an die kantonalen kirchlichen Körperschaften.»; § 19 Abs. 2 KiG-ZH: «Er unterstützt mit den Kostenbeiträgen ihre Tätigkeiten mit Bedeutung für die ganze Gesellschaft, insbesondere in den Bereichen Bildung, Soziales und Kultur.»

16 Verfassung des Kantons Zürich vom 27. Februar 2005 (LS 101; SR 131.211).

17 Das Zürcher Kirchengesetz enthält eine Übergangsbestimmung, welche den Gesamtbetrag der Kostenbeiträge zugunsten der kirchlichen Körperschaften für die erste Beitragsperiode (20102013) auf 50 Mio. Fr. pro Jahr festlegt: § 29 Abs. $1 \mathrm{KiG}-\mathrm{ZH}$. Diese Summe wurde für die erste

\section{Finanzrechtliche Einordnung}

8 Hinsichtlich der finanzrechtlichen Einordnung der staatlichen Leistungen ist vor allem zwischen Abgeltungen und Finanzhilfen (Subventionen) zu unterscheiden: Mit Abgeltungen wird die Erfüllung einer Staatsaufgabe durch nichtstaatliche Träger entschädigt ${ }^{18}$. Diese Unterstützung macht bei der staatlichen Finanzierung von Kirchen und Religionsgemeinschaften nur einen kleinen Teil aus ${ }^{19}$. Wichtiger sind im vorliegenden Zusammenhang allgemeine Zuwendungen, die am ehesten als Finanzhilfen bzw. Subventionen zu kategorisieren sind $^{20}$. Damit wird nicht die Erfüllung einer bestimmten Staatsaufgabe durch Kirchen abgegolten, sondern deren eigene Tätigkeit unterstützt.

9 Die Leistungen des Staates an die Kirchen lassen sich zum grössten Teil dem Bereich der Finanzhilfen zuordnen, wobei gewisse Vorbehalte anzubringen sind. So leistet der Kanton Zürich zugunsten der Kirchen sogenannte «Kostenbeiträge», die er von Subventionen abgrenzt. Die Kostenbeiträge, so der Regierungsrat in der Weisung zum Kirchengesetz von 2007, enthielten Elemente sowohl von Kostenanteilen als auch von Subventionen. Wie die Ersteren würden sie den Bezügern einen rechtlichen Anspruch

reguläre Beitragsperiode (2014-2019) beibehalten.

18 Hafner/Ebnöther (Fn. 8), S. $151 \mathrm{f}$.

19 Michael Marti/Eliane Kraft/Felix Walter, Dienstleistungen, Nutzen und Finanzierung von Religionsgemeinschaften in der Schweiz. Synthese des Projekts FAKIR (Finanzanalyse Kirchen) im Rahmen des NFP 58 «Religionsgemeinschaften, Staat und Gesellschaft», Glarus/Chur 2010, S. 33.

20 Vgl. Cavelti/Kley (Fn. 12), Rz. 38 ff.; Daniel Kosch, Die öffentliche Finanzierung der katholischen Kirche in der Schweiz. Zahlen, Zusammenhänge und Zukunftsperspektiven, Zürich 2013, S. 30 . 
verleihen, der jedoch ähnlich wie die Subventionen im Einzelnen noch genauer festzulegen sei $^{21}$.

10 Echte finanzielle Fördermassnahmen sind nur die Finanzhilfen ${ }^{22}$. Bei den anderen Ausgaben zugunsten der Kirchen (Abgeltungen) werden ja Leistungen entschädigt, die der Staat ohnehin erbringen müsste. Die vorliegende Darstellung konzentriert sich daher auf die Finanzhilfen zugunsten der Religionsgemeinschaften.

\section{Bezug zum Anerkennungssystem}

11 Für das Verhältnis zwischen Staat und Religionsgemeinschaften ist das Institut der öffentlich-rechtlichen Anerkennung zentral. Einzelne Religionsgemeinschaften werden in der Form öffentlichrechtlicher Körperschaften staatlich anerkannt. Mit diesem Status gehen verschiedene Privilegien einher, wie etwa der Zugang zur Seelsorgetätigkeit in öffentlichen Spitälern oder die Möglichkeit, in Schulen Religionsunterricht anzubieten²3. Die finanzielle Förderung durch staatliche Beiträge, die nicht Abgeltungen für konkrete Beiträge sind, gehört zu

21 Antrag und Weisung zum Kirchengesetz vom 31. Mai 2006, Amtsblatt 2006, S. 573 ff., 595. Genau betrachtet, entsprechen die Beiträge an die anerkannten Religionsgemeinschaften allerdings auch nicht genau dem Begriff der Kostenbeiträge gemäss dem Staatsbeitragsgesetz des Kantons Zürich. Diesem zufolge sind Kostenbeiträge Staatsbeiträge, auf die das Gesetz einen Anspruch einräumt (§ 2a des Staatsbeitragsgesetzes vom 1. April 1990 [LS 132.2]). Kostenbeiträge gelten daher als gebundene Ausgaben im Sinn von $\S 37$ Abs. 2 des Gesetzes über Controlling und Rechnungslegung vom 9. Januar 2006 (CRG; LS 611). Über gebundene Ausgaben kann der Regierungsrat selbst entscheiden (vgl. § 36 CRG). Dagegen entscheidet über die Kostenbeiträge zugunsten der anerkannten Religionsgemeinschaften der Kantonsrat (§ $20 \mathrm{KiG}-\mathrm{ZH}$ ).

${ }_{22}$ Hafner/Ebnöther (Fn. 8), S. 158.

23 Vgl. Engi (Fn. 2), S. 212 f., mit weiteren Nachweisen. den typischen Anerkennungswirkungen. Soweit Kantone die Anerkennung vorsehen, fliessen staatliche Leistungen dieser Art daher allein an die anerkannten Religionsgemeinschaften.

In Ausnahmefällen ist die finanzielle Unterstützung nicht an die Anerkennung geknüpft24. So gibt es im Kanton Neuenburg - wie im Kanton Genf - keine öffentlich-rechtliche Anerkennung von Religionsgemeinschaften. Einige Religionsgemeinschaften sind aber als «institution d'interêt public» anerkannt ${ }^{25}$. Der Kanton unterstützt die Leistungen, die diese Gemeinschaften zugunsten der Gesellschaft leisten ${ }^{26}$.

13 Neben der öffentlich-rechtlichen Anerkennung gibt es in vielen Kantonen Formen der sogenannten kleinen Anerkennung. Bei dieser bleiben die anerkannten Religionsgemeinschaften privatrechtliche Vereine, werden also nicht zu Körperschaften des öffentlichen Rechts ${ }^{27}$. In manchen Kantonen sind beispielsweise jüdische Gemeinschaften in dieser Form anerkannt. Bezüglich der staatlichen finanziellen Unterstützung ist die Situation bei dieser Anerkennungsform unterschiedlich. Teilweise partizipieren auch Gemeinschaften, die in der «kleinen» Form anerkannt sind, an staatlichen Beiträgen. Das ist zum Beispiel im Kanton Zürich bezüglich der anerkannten jüdischen Gemeinden der Fall ${ }^{28}$. In anderen

$24 \overline{\text { Cla Reto Famos, Die öffentlichrechtliche Aner- }}$ kennung von Religionsgemeinschaften im Lichte des Rechtsgleichheitsprinzips, Diss. St. Gallen 1999, S. 44.

25 Art. 98 Abs. 1 KV NE.

26 Art. 98 Abs. 3 KV NE.

$27 \mathrm{Zu}$ den verschiedenen Formen der Anerkennung Engi (Fn. 2), S. 217-219.

28 \& 8 Gesetz über die jüdischen Gemeinden vom 9. Juli 2007 (LS 184.1). 
Fällen werden diese Gemeinschaften dagegen finanziell nicht unterstützt. So erhalten die Gemeinschaften, die im Kanton Basel-Stadt kantonal anerkannt sind $^{29}$, keine staatlichen Gelder ${ }^{30}$.

\section{Praxis}

\section{Allgemeine Grössenordnung}

14 Der Gesamtumfang der staatlichen Leistungen zugunsten der Kirchen und Religionsgemeinschaften beträgt nach neueren Erhebungen (2010) gesamtschweizerisch etwa 292 Mio. Fr. pro Jahr31. Für die katholische Kirche wurden die Beiträge der öffentlichen Hand ausserhalb der Kirchensteuern im Jahr 2013 mit 110 Mio. Fr. beziffert32. Die staatlichen Beiträge gehen weit überwiegend an die beiden grossen christlichen Kirchen, die evangelisch-reformierte und die römischkatholische (im Fall der letzteren an die jeweilige kantonale Körperschaft).

29 Gem. § 133 f. KV BS.

30 Es handelt sich aktuell um zwei alevitische Vereinigungen, die Christengemeinschaft und die Neuapostolische Kirche. Die Rechte und Pflichten werden bei dieser Anerkennungsform im Einzelfall festgelegt ( 133 Abs. 4 KV BS). Vgl. Christoph Winzeler, Die neue Anerkennungspraxis im Religionsverfassungsrecht des Kantons BaselStadt, in: René Pahud de Mortanges (Hrsg.), Staatliche Anerkennung von Religionsgemeinschaften. Zukunfts- oder Auslaufmodell?, Zürich 2015, S. 25-37 (zum symbolischen Gehalt der bisherigen kantonalen Anerkennungen insb. S. 32 f.).

31 Marti/Kraft/Walter (Fn. 19), S. 67. Total 556 Mio. Fr. Staatsbeiträge und Steuern juristischer Personen für die evang.-ref. und die röm.-kath. Kirche, davon 264 Mio. Fr. aus den Steuern der juristischen Personen.

32 Kosch (Fn. 20), S. 12.

\section{Keine oder nur geringe Staatsbeiträge}

15 Eine Reihe von Kantonen kennt kein System allgemeiner Staatsbeiträge für Religionsgemeinschaften. Es finden sich keine staatlichen Normen dazu, oder es ist wo solche Grundlagen bestehen - nach den offiziellen Dokumenten kein Geldfluss feststellbar. Zu dieser Gruppe gehören:

- Genf

- Aargau33

- Obwalden

- Nidwalden

- Luzern

- Schwyz

- Appenzell Innerrhoden

- Zug

- Uri

- Glarus 34

16 Gemäss einer Studie des Büros Ecoplan aus dem Jahr 2010 gibt es in vier dieser Kantone eine öffentliche Finanzierung ausserhalb der Kirchensteuern in kleinerem Umfang: Luzern (o,3 Mio. Fr.), Obwalden (o,6 Mio. Fr.), Uri (o,2 Mio. Fr.), Zug (0,1 Mio. Fr.)35. Es handelt sich bei diesen Geldern um kleinere Staatsbeiträge für definierte Leistungen 36 .

33 Vgl. Reformierte Landeskirche Aargau, Dokument «Trennung von Kirche und Staat im Kanton Aaargau. Finanzierung der Landeskirche».

34 Gemäss der Glarner Kantonsverfassung kann der Kanton die überkonfessionellen öffentlichen Arbeiten der Kirchen mit Beiträgen unterstützen; Art. 137 Abs. 2 KV GL; vgl. Art. 31 Abs. 1 lit. d Verfassung der Evangelisch-Reformierten Landeskirche des Kantons Glarus vom 27. Juni 1990 (GS IV A/1/4).

35 Marti/Kraft/Walter (Fn. 19), S. 77 f.

36 Schriftliche Auskunft von Dr. Michael Marti vom 12. Dezember 2017. 


\section{Hohe Beiträge in drei Kantonen}

17 Drei Kantone der Schweiz sind in besonderer Weise von einer Vergangenheit des Staatskirchentums geprägt: Bern, Waadt und Zürich. Die jeweilige reformierte Kirche war in diesen Kantonen über lange Zeit die eigentliche und einzige offizielle Kirche. Im Kanton Waadt hatte die reformierte Kirche bis zur jüngsten Verfassungsänderung (2002) den Rang einer «institution national»37. In allen drei Kantonen wurden Pfarrstellen lange Zeit staatlich finanziert, und teilweise ist das heute noch der Fall. Dementsprechend kennen diese drei Kantone bis heute hohe staatliche Beiträge an die anerkannten Kirchen.

- Im Kanton Bern trägt der Kanton die Besoldung der Pfarrstellen ${ }^{8}$. Unlängst wurde eine Revision des Landeskirchengesetzes beschlossen, die Änderungen im Finanzierungssystem bringt39. Künftig wird nur noch ein Teil der Pfarrstellen aufgrund historischer Rechtstitel durch den Staat finanziert werden, ein anderer Teil der Unterstützung wird aufgrund gesamtgesellschaftlich relevanter Leistungen erfolgen ${ }^{\circ}$. Insgesamt sollen

37 Art. 13 der Kantonsverfassung von 1885.

38 Vgl. Art. 19 Abs. 1 Gesetz über die bernischen Landeskirchen vom 6. Mai 1945 (BSG 410.11).

39 Das neue Gesetz über die bernischen Landeskirchen wurde vom Grossen Rat am 21. März 2018 mit 118 Ja- und 10 Nein-Stimmen bei 8 Enthaltungen angenommen. Die Referendumsfrist läuft bis zum 18. Juli 2018. Das Gesetz soll am 1. Januar 2020 in Kraft treten.

40 Gesetz über die Landeskirchen (Landeskirchengesetz, LKG), Antrag des Regierungsrates, 29. März 2017, S. 11 ff. Zum Hintergrund der historischen Rechtstitel im Kanton Bern Christina Schmid-Tschirren (Fn. 13), insb. S. 165 ff., 425 ff.; Kaspar Sutter/Markus Müller, Historische Rechtspositionen. Fortwirkung oder Untergang? Überlegungen am Beispiel der staatlichen Pfarrerbesoldung im Kanton Bern, in: Schweizeri- aber staatliche Beiträge in gleicher Höhe wie bisher bezahlt werden. Gemäss der Jahresrechnung 2016 des Kantons Bern wurden 73 Mio. Fr. für die pfarramtliche Versorgung der Kirchgemeinden und Beziehungen zwischen Kirche und Staat aufgewen$\operatorname{det} 41$.

- Im Kanton Waadt gibt es eine weitgehende staatliche Unterstützung der Kirchen. Hingegen werden keine Kirchensteuern erhoben ${ }^{42}$. Nach waadtländischem Recht leistet der Kanton die Unterstützung, die nötig ist, damit die anerkannten Kirchen ihre «mission au service du tous» leisten können43. Dazu wird ein Vertrag zwischen Staat und Kirchen geschlossen 44. Insgesamt beträgt die finanzielle Unterstützung für die Kirchen aktuell (2014) gut 61 Mio. Franken45.

sches Zentralblatt für Staats- und Verwaltungsrecht 114 (2013), S. 471-490.

41 Geschäftsbericht mit Jahresrechnung 2016 des Kantons Bern, Band 1, S. 82. Vgl. auch «Kirche und Staat unterwegs zu einer neuen Partnerschaft», Medienmitteilung des Kantons Bern und der Evangelisch-reformierten Landeskirche vom 8. Dezember 2015. In der Presse ist von rund 75 Mio. Fr. die Rede: Berner Zeitung, «Biblische Diskussion um null Franken», bernerzeitung.ch, 7. September 2017.

42 Christoph Winzeler, Einführung in das Religionsverfassungsrecht der Schweiz, 2. Aufl., Zürich/Basel/Genf 2009, S. 104.

43 Art. 170 Abs. 2 der Verfassung des Kantons Waadt vom 14. April 2015 (SR 131.231); Art. 13 Abs. 1 Loi sur les relations entre l'Etat et les Eglises reconnues de droit public (LREEDP) du 9 janvier 2007.

44 Art. 13 Abs. 2 LREEDP.

45 Eglise évangélique réformée du canton de Vaud, Pressemitteilung, 30. September 2014. Nach einer anderen Quelle wurden 2002 29,5 Mio. Fr. Pfarrgehälter und 8,3 Mio. Fr. Subventionen an die evangelisch-reformierte Kirche gezahlt: Stefan Streiff, Kirchenfinanzen in der pluralistischen Gesellschaft. Die Einnahmen reformierter Kirchen in der Schweiz aus theologischer Perspektive, Zürich/Basel/Genf 2008, S. 77. 
- Der Kanton Zürich hat sein System der Kirchenfinanzierung mit dem Kirchengesetz von 2007 umgestellt. Bildeten früher historische Rechtsansprüche die Grundlage der Finanzierung, sind es nunmehr Leistungen von gesamtgesellschaftlicher Bedeutung, insbesondere in den Bereichen Bildung, Soziales und Kultur46. Auf dieser Grundlage werden die Leistungen zugunsten der anerkannten Körperschaften für eine Beitragsperiode von jeweils sechs Jahren in Form eines Globalkredits vom Kantonsrat beschlossen47. In der aktuellen Periode 2014-2019 beträgt der Gesamtbetrag 300 Mio. Fr., 50 Mio. Fr. pro Jahr ${ }^{48}$.

18 Gemäss diesen Angaben beträgt die öffentliche Unterstützung der Kirchen aus allgemeinen Steuermitteln (ausserhalb der Kirchensteuern) in den Kantonen Bern, Waadt und Zürich insgesamt etwa 184 Mio. Fr. Gemessen am Gesamtvolumen der Finanzierung in allen Kantonen von etwa 292 Mio. Fr. (vorn Ziff. III.1) ist das knapp zwei Drittel. Dies unterstreicht die besondere Rolle dieser drei Kantone mit ihrer speziellen, reformierten Tradition im System der finanziellen Beziehungen zwischen Staat und Religionsgemeinschaften.

\section{Bezifferte Beträge}

19 Einige Kantone legen den Beitrag für die anerkannten Kirchen in ihren Gesetzen ziffernmässig fest:

§19 Abs. 2 KiG-ZH.

$47 \S 20$ Abs. $1 \mathrm{KiG}-\mathrm{ZH}$.

48 Beschluss des Kantonsrates vom 3. Dezember 2012, 4927/2012 Bewilligung eines Rahmenkredits für die Kostenbeiträge an die anerkannten Religionsgemeinschaften für die Beitragsperiode 2014-2019.
- Im Kanton Basel-Landschaft bestehen die Beiträge an die Kirchen 49 aus einem jährlichen Grundbetrag von 100'ooo Fr. und einem Beitrag von 35 Franken pro Kirchenmitglied50 (wobei der Betrag indexiert ist $\left.{ }^{11}\right)$.

- Im Kanton Schaffhausen ist gesetzlich ein jährlicher Beitrag von 3,7 Mio. Fr. an die Landeskirchen vorgesehen. Dieser Betrag entspricht dem Stand des Landesindexes der Konsumentenpreise vom November 2014 und wird jährlich der Entwicklung dieses Indexes angepasst 52 .

- Im Kanton Neuenburg werden - wie bereits erwähnt - einige Religionsgemeinschaften als «institutions d'interêt public» anerkannt53. Deren Leistungen zugunsten der Gemeinschaft werden staatlich unterstützt54. Der entsprechende Vertrag sieht zurzeit Zahlungen von 1,5 Mio. Fr. pro Jahr vor55.

\section{Autonomie der Gemeinden}

2o In einzelnen Kantonen ist die Finanzierung von Religionsgemeinschaften teilweise den Gemeinden überlassen, so dass innerhalb des Kantons verschiedene Modelle bestehen:

$49 \overline{\text { Gesetzliche Grundlage: § } 8 \text { Abs. } 1 \text { lit. c Kirchenge- }}$ setz vom 3. April 1950 (SGS 191; nachfolgend: Kirchengesetz Basel-Landschaft).

50 § 8c Abs. 2 Kirchengesetz Basel-Landschaft.

$51 \quad$ § 8c Abs. 4 Kirchengesetz Basel-Landschaft.

52 Art. 1 Abs. 1 Gesetz über die Ausrichtung von Beiträgen an die Landeskirchen vom 22. November 1982 (SHR 130.100).

53 Vorn Kap. II.3.

54 Art. 98 Abs. 1 KV NE.

55 Art. 4 Concordat entre l'Etat de Neuchâtel et l'Eglise réformée évangelique du Canton de Neuchâtel, l'Eglise catholique romaine, l'Eglise catholique chrétienne du 2 mai 2001. 
- Im Kanton Wallis tragen die politischen Gemeinden die Kosten, die die Pfarreien nicht zu tragen vermögen. Der Kanton erstattet einen Staatsbeitrag an die Diözese ${ }^{5}$. Der Umfang der öffentlichen Finanzierung ist vergleichsweise hoch, sie beträgt gemäss der Studie von Ecoplan (vorn Kap. III.2) etwa 27 Mio. Fr.57

- Im Tessin ist die Finanzierung den einzelnen Gemeinden überlassen 58 . Nach den verfügbaren Zahlen ist die Finanzierung eher gering, allerdings gibt es zur Unterstützung der römisch-katholischen Kirche keine verlässlichen Angaben für den ganzen Kanton. 59

\section{Weitere Systeme, insbesondere Unterstützung bestimmter Leistungen}

21 In den übrigen acht Kantonen bestehen verschiedene Systeme. Häufig ist vorgesehen, dass der Staat bestimmte Leistungen der Kirchen unterstützen kann.

- In Freiburg sieht das Gesetz vor, dass der Staat Beiträge leistet für die Erfüllung von sozialen und karitativen Aufgaben sowie Aufgaben in der Ausbildung, für den Bau und Ausbau von Gebäuden und Einrichtungen, die nicht vorwiegend einem religiösen Ziel dienen, für die Ausübung der Seelsorge in staatlichen Anstalten, sowie in anderen spezialgesetzlich vorgesehenen Fällen, namentlich im

${ }_{56}$ Kosch (Fn. 20), S. 26.

Vgl. Marti/Kraft/Walter (Fn. 19), S. 77 f.

Kosch (Fn. 20), S. 26.

59 Vgl. Marti/Kraft/Walter (Fn. 19), S. 77 f.
Bereich des Kulturgüterschutzes ${ }^{60}$. Die Beiträge sind eher gering und haben einen Umfang von zirka 0,7 Mio. Fr. ${ }^{61}$

- Im Kanton Graubünden werden Leistungen in den Bereichen Spital- und Gefängnisseelsorge und Denkmalschutz unterstützt ${ }^{62}$. Der Umfang der öffentlichen Finanzierung beträgt etwa o,6 Mio. Fr.

- Im Kanton Appenzell Ausserrhoden sind Staatsbeiträge für Gefängnisund Spitalseelsorge möglich63.

- Der Kanton St. Gallen leistet Staatsbeiträge an die Spital- und Gefängnisseelsorge ${ }^{64}$. Der Umfang der öffentlichen Finanzierung ist relativ hoch, gemäss Studien beträgt er für die reformierte und die katholische Kirche zusammen 37,9 Mio. Fr. 65

- Der Kanton Basel-Stadt leistet gemäss Kirchengesetz staatliche Unterstützung für die Seelsorge in Spitälern, Heimen und Gefängnissen, sowie im Bereich der Bauten ${ }^{66}$. Gemäss Anga-

$60 \overline{\text { Art. } 22 \text { Gesetz über die Beziehungen zwischen }}$ den Kirchen und dem Staat vom 16. September 1990 (SGF 190.1).

61 Marti/Kraft/Walter (Fn. 19), S. 77 f.

62 Botschaft der Regierung an den Grossen Rat, Bericht zur Finanzierung der Kirchen im Kanton Graubünden, Heft Nr. 8/2015-2016, S. 527 ff., $540 \mathrm{f}$.

63 Römisch-katholische Zentralkonferenz der Schweiz (Hrsg.), Kirche und Staat in der Schweiz. Übersicht über die Situation in den einzelnen Diözesen und Kantonen, Zürich 2010, S. 34.

64 Kirche und Staat in der Schweiz (Fn. 63), S. 33.

65 Marti/Kraft/Walter (Fn. 19), S. 77 f. Darauf gestützt auch Kosch (Fn. 20), S. 13: öffentliche Finanzierung der katholischen Kirche im Kanton St. Gallen ausserhalb der Kirchensteuern 27,2 Mio. Fr.

$66 \S \S 8-12$ Gesetz betreffend die Staatsoberaufsicht über die öffentlich-rechtlichen Kirchen und die Israelitische Gemeinde sowie über die Verwen- 
ben aus der Literatur betrug die staatliche Unterstützung für die reformierte Kirche 2002 0,5 Mio. Fr. ${ }^{67}$

- Der Kanton Solothurn leistet Beiträge etwa in Form einer Wohnungsentschädigung an den Bischof von Basel, eines Verwaltungs- und Besoldungsbeitrags für den Weihbischof etc. 68 Die Förderung der reformierten und katholischen Kirche hat einen Umfang von zirka 5 Mio. Fr. 69

- Im Kanton Jura erhalten die anerkannten Religionsgemeinschaften einen jährlichen Beitrag, der sich nach den Lohnkosten dieser Gemeinschaften bemisst70. Die Unterstützung beträgt zwischen 45 und $55 \%$ der jährlichen Lohnsumme ${ }^{71}$. Darüber hinaus kann der Kanton bestimmte Tätigkeiten der Kirchen unterstützen und zum Beispiel Baubeiträge sprechen72.

- Für den Kanton Thurgau weisen empirische Untersuchungen ebenfalls staatliche Leistungen in einem geringeren Umfang nach73. Weiterführende Informationen dazu sind nicht verfügbar.

dung von Staats- und Gemeindemitteln zu Kirchenzwecken (Kirchengesetz) vom 8. November 1973 (SG 190.100).

67 Streiff (Fn. 45), S. 39.

68 Geschäftsbericht: Finanzen und Leistungen, Botschaft und Entwurf des Regierungsrates an den Kantonsrat von Solothurn vom 27. März 2018, RRB Nr. 2018/458.

69 Marti/Kraft/Walter (Fn. 19), S. 77 f.

70 Art. 26 Loi concernant les rapports entre les Eglises et l'Etat du 26 octobre 1978 (RSJU 471.1).

71 Art. 26 Abs. 4 Loi concernant les rapports entre les Eglises et l'Etat du 26 octobre 1978.

72 Art. 27 Loi concernant les rapports entre les Eglises et l'Etat du 26 octobre 1978.

73 Marti/Kraft/Walter (Fn. 19), S. 77.

\section{Problematik}

22 Die bisherige Darstellung zeigt einen beträchtlichen Umfang der öffentlichen Finanzierung von Religionsgemeinschaften. Heute werden diese Leistungen überwiegend mit Leistungen der anerkannten Religionsgemeinschaften von gesamtgesellschaftlicher Bedeutung begründet. Namentlich in den drei Kantonen, in denen die Beiträge am höchsten sind, steht heute diese Begründung im Vordergrund. Dazu wurde bereits einiges bemerkt, doch soll dies noch etwas genauer betrachtet werden.

23 Der Kanton Zürich umschreibt die Grundlagen der staatlichen Leistungen in $\S 19$ Abs. 2 des Kirchengesetzes folgendermassen74: «Er [der Kanton] unterstützt mit den Kostenbeiträgen ihre Tätigkeiten [die Tätigkeiten der kantonalen kirchlichen Körperschaften] mit Bedeutung für die ganze Gesellschaft, insbesondere in den Bereichen Bildung, Soziales und Kultur.» Um die Beiträge zu erhalten, müssen die kirchlichen Körperschaften Programme zur Erbringung von Tätigkeiten mit gesamtgesellschaftlicher Bedeutung erstellen75.

24 Der Kanton Bern hat im Zuge der Revision des Kirchengesetzes zusätzlich zu den historischen Rechtstiteln eine zweite Säule zur Kirchenfinanzierung geschaffen. Dabei wird ein Teil der staatlichen Leistungen durch kirchliche Tätigkeiten von gesamtgesellschaftlicher Bedeutung begründet. Art. 31 Abs. 1 des neuen Landeskirchengesetzes schreibt vor: «Mit einem Beitrag unterstützt der Kanton die Landeskirchen für die von ihnen im ge-

74 Dazu schon oben Fn. 15.

$75 \S 19$ Abs. 3 KiG-ZH. 
samtgesellschaftlichen Interesse nach Artikel 3 erbrachten Leistungen.»76 Als Leistungen im gesamtgesellschaftlichen Interesse nennt das Gesetz unter anderem: Kinder- und Jugendarbeit; Angebote $\mathrm{zu}$ Ehe, Familie und Partnerschaft; Erwachsenenbildung; Angebote für Migrantinnen und Migranten sowie Asylsuchende; Kultur sowie Seelsorge77.

Der Kanton Waadt gewährt den anerkannten Kirchen gemäss Verfassung «die notwendigen Mittel zur Erfüllung der Aufgaben, die sie im Kanton im Dienste aller wahrnehmen.»78 Auch in diesem Fall bilden somit die Leistungen zugunsten der Gesamtgesellschaft die Legitimationsgrundlage der Unterstützung79.

Die sozialen Leistungen der Kirchen wurden schon mehrfach empirisch erhoben $^{80}$. Dabei zeigte sich, dass die anerkannten Religionsgemeinschaften Tätig-

76 Art. 3 Abs. 1 LKG: «Die Landeskirchen tragen im gesamtgesellschaftlichen Interesse zur solidarischen Gemeinschaft, zur Vermittlung grundlegender Werte, zum Frieden unter den Religionen, zur religiösen Bildung und zur Kulturpflege bei.»

77 Art. 31 Abs. 2 LKG.

78 Art. 170 Abs. 2 KV-VD, dazu schon oben Fn. 43.

79 Einen Verweis auf historische Rechtsansprüche enthält zum Beispiel noch das Gesetz im Kanton Schaffhausen; vgl. Art. 1 Abs. 2 Gesetz über die Ausrichtung von Beiträgen an die Landeskirchen vom 22. November 1982 (SHR 130.100): «Diese Leistung erfolgt zum Teil aufgrund von historischen Rechtstiteln.»

80 Vgl. u.a. Charles Landert, Die Leistungen der Reformierten Kirchen Bern-Jura in Diakonie und Beratung, Bildung und Kultur, Bern 2000; ders., Die Neuordnung des Verhältnisses zwischen dem Kanton und den öffentlich-rechtlich anerkannten Kirchen und Wege der Finanzierung kirchlicher Leistungen, Zürich 1999; ders., Die sozialen und kulturellen Leistungen der Evangelischreformierten Landeskirche des Kantons Zürich, Zürich 1995; Fachhochschule Nordwestschweiz, Die freiwilligen sozialen Leistungen der Kirchen im Kanton Solothurn. Ergebnisse einer Befragung der Kirchgemeinden und kirchlichen Dienststellen im Kanton Solothurn, Olten 2007. keiten mit gesamtgesellschaftlicher Bedeutung erbringen, die den Umfang der staatlichen Unterstützung zumindest decken, tendenziell übersteigen. Eine Studie, die kürzlich im Kanton Zürich durchgeführt wurde und den Begriff der «Tätigkeiten mit Bedeutung für die ganze Gesellschaft» auf sozialwissenschaftlicher Grundlage relativ eng fasste, brachte ebenfalls entsprechende Ergebnisse ${ }^{81}$.

27 Wird die staatliche Unterstützung mit den gesamtgesellschaftlich relevanten Leistungen der Religionsgemeinschaften begründet, bringt das verschiedene Vorteile. Insbesondere steht eine rein säkulare Begründung zur Verfügung, die nicht in irgendeiner Weise auf religiöse oder theologische Inhalte Bezug nehmen muss $^{82}$. Dieser Legitimationsmodus führt aber auch eine gewichtige Problematik mit sich. Es ist anzunehmen, dass auch nicht-anerkannte Religionsgemeinschaften Leistungen von gesamtgesellschaftlicher Bedeutung erbringen. Systematisch erforscht wurde das bisher noch nicht. Ohne Zweifel wäre der Umfang - etwa bei christlich-orthodoxen oder muslimischen Gemeinschaften - dieser Leistungen viel geringer als namentlich bei den beiden grossen christlichen Kirchen. Es ist jedoch hoch wahrscheinlich, dass Leistungen dieser Art bei nichtanerkannten Religionsgemeinschaften ebenfalls nachzuweisen wären.

81 Thomas Widmer u.a., Kirchliche Tätigkeiten mit gesamtgesellschaftlicher Bedeutung im Kanton Zürich, Zürcher Politik- und Evaluationsstudien Nr. 18, Juni 2017.

82 Im Rahmen dieses Aufsatzes gehe ich nicht näher auf die Sonderthematik ein, dass das Wirken der Kirchen gewissermassen verkürzt betrachtet wird, wenn es allein unter dem Aspekt sozial erwünschter Leistungen gewürdigt wird; vgl. dazu Engi (Fn. 2), S. 242 f., mit weiteren Nachweisen. 

genwärtig an das System der öffentlichrechtlichen Anerkennung gebunden. Den nicht-anerkannten Religionsgemeinschaften ist der Zugang zu dieser Art der Finanzierung verwehrt. Der Weg zu neuen Anerkennungen ist gleichzeitig sehr hürdenreich. Das Verfahren der öffentlich-rechtlichen Anerkennung ist in den Kantonen politisch bestimmt. In der Regel sind für Neuanerkennungen Verfassungsänderungen nötig, die ein obligatorisches Referendum verlangen; die Möglichkeit zumindest des fakultativen Referendums besteht fast immer ${ }^{83}$. Klar definierte Voraussetzungen für neue Anerkennungen bestehen nur in einer Minderheit der Kantone, und auch dort häufig nur in Bezug auf die sogenannte kleine Anerkennung ${ }^{84}$. Dazu kommen viele weitere Probleme, namentlich im Hinblick auf den Organisationsgrad der Religionsgemeinschaften, die potenziell anerkannt werden könnten ${ }^{85}$. Unter diesen Bedingungen ist es zumindest auf kürzere Sicht kaum realistisch, dass die Ungleichheiten bezüglich der öffentlichen Finanzierung im Wege neuer Anerkennungen abgebaut werden können.

29 Dies führt zur Situation, dass Leistungen von gesamtgesellschaftlicher Bedeutung bei einigen Religionsgemeinschaften den anerkannten - staatlich unterstützt werden, bei anderen aber nicht. Es wird

$8_{3}$ Regina Kiener/Sebastian Kempe, Zwischen Tradition und Grundrechtsschutz: Die öffentlichrechtliche Anerkennung von Glaubensgemeinschaften, in: Schweizerisches Zentralblatt für Staats- und Verwaltungsrecht 117 (2016), S. 567$587,572 \mathrm{ff}$.

84 Lorenz Engi, Islamische Religionsgemeinschaften öffentlich-rechtlich anerkennen?, in: Aktuelle Juristische Praxis 26 (2017), S. 1210-1221, 1213; Kiener/Kempe (Fn. 83), S. 573 f.

85 Engi (Fn. 84), S. $1217 \mathrm{f}$. eine neue Begründung verwendet (gesellschaftlich relevante Leistungen), diese wird aber nicht gleichermassen auf alle in Betracht kommenden Gemeinschaften angewandt. Während die entsprechende Grenzziehung beim Begründungsmodus der historischen Rechtstitel trägt, ist sie bei der Begründung über gesamtgesellschaftlich bedeutende Leistungen kaum möglich. Man ist gewissermassen den ersten Schritt gegangen - Begründung über die sozial relevanten Leistungen aber noch nicht den zweiten: Unterstützung solcher Leistungen bei allen Religionsgemeinschaften.

30 Bis zu einem gewissen Grad ist diese Ungleichbehandlung damit zu rechtfertigen, dass der Anerkennungsstatus gerade diese Vorrechte vermittelt. Auf Dauer dürfte es aus Rechtsgleichheits- und Neutralitätsgründen jedoch problematisch werden, die Finanzierung in dieser Weise zu beschränken. Daher sind Möglichkeiten der Weiterentwicklung zu prüfen. Einige Tendenzen in der Praxis, die in diese Richtung gehen, seien abschliessend näher betrachtet.

\section{Zukunftsperspektiven}

31 In der Literatur und in der Praxis mehren sich Stimmen, die eine Einbeziehung nicht-anerkannter Gemeinschaften in das Finanzierungssystem zur Diskussion stellen. Das System der Staatsbeiträge wird in seiner bestehenden Form zunehmend hinterfragt. So wird in den Staatsbeiträgen in neueren Stellungnahmen etwa eine Privilegierung gesehen, die den pluralistischen Verhältnissen nur sehr bedingt adäquat sei ${ }^{86}$. Nach anderen Stimmen drängt es sich auf, nicht nur die

86 Streiff (Fn. 45), S. 87. 
Ungleichbehandlung von Glaubensgemeinschaften untereinander, sondern die Besserstellung von Glaubensgemeinschaften gegenüber anderen Vereinigungen mit ideellem Zweck zu hinterfragen ${ }^{87}$. Aus dem kirchlichen Bereich wird festgehalten, dass eine Berücksichtigung von anderen als den öffentlich-rechtlich anerkannten Religionsgemeinschaften bei der Finanzierung grundsätzlich möglich sei. Die öffentliche Hand unterstütze viele Institutionen, die keinen Körperschaftsstatus hätten 88 .

Dem entsprechen bestimmte Tendenzen in der Praxis. An verschiedenen Stellen werden Ansätze sichtbar, das bestehende Finanzierungssystem weiterzuentwickeln:

- Der Regierungsrat des Kantons Bern verabschiedete im Hinblick auf die Revision des Kirchengesetzes acht Leitsätze, die später auch vom Grossen Rat (als «Planungserklärungen») übernommen wurden. Leitsatz 8 lautet: "Auf die Ausarbeitung eines allgemeinen Anerkennungsgesetzes wird bis auf weiteres verzichtet. Anstelle von Anerkennungen sind andere Massnahmen zur Förderung von Religionsgemeinschaften, die gesellschaftlich relevante Leistungen erbringen, zu prüfen.» 89

- Der Regierungsrat des Kantons Zürich veröffentlichte im vergangenen Jahr ein Orientierungspapier zum Verhältnis zwischen Staat und Religion ${ }^{90}$. In sieben Leitsätzen legte er sei-

87 Kiener/Kempe (Fn. 83), S. 587.

88 Kosch (Fn. 20), S. 37.

89 Regierungsrat Bern, Acht Leitsätze als Basis für die Totalrevision des Kirchengesetzes, 27. März 2015.

90 Staat und Religion im Kanton Zürich. Eine Orientierung, 2017. ne grundsätzliche Haltung dazu dar, wobei Leitsatz 7 lautet: «Zum Umgang mit verfassungsrechtlich nichtanerkannten Religionsgemeinschaften braucht es klare Handlungsgrundlagen.» In der Erklärung zu diesem Leitsatz wird ebenfalls die Frage einer öffentlichen Finanzierung von Leistungen nicht-anerkannter Gemeinschaften thematisiert.

- In der erwähnten Studie, die im Kanton Zürich unlängst zu kirchlichen Tätigkeiten mit gesamtgesellschaftlicher Bedeutung durchgeführt wurden, werden Empfehlungen abgegeben. Eine davon lautet: «Je nach weiterer Entwicklung im Bereich der Anerkennung von anderen Glaubensgemeinschaften ist mittelfristig zu prüfen, inwiefern sich andere religiöse Gemeinschaften ebenfalls für Staatsbeiträge der Art, wie sie an die Landeskirchen gehen, qualifizieren (können).»91

33 Seit Längerem ist eine Abkoppelung von Wirkungen der Anerkennung vom Status der öffentlich-rechtlichen Anerkennung zu beobachten ${ }^{92}$. So üben heute beispielsweise auch nicht-anerkannte Religionsgemeinschaften in vielen Kantonen Seelsorge in öffentlichen Institutionen (namentlich in Spitälern) aus. Diese Möglichkeit bildet eigentlich eine klassische Anerkennungsfolge, die heute aber - im Wesentlichen einfach aufgrund des faktischen Bedarfs - unabhängig von der

91 Thomas Widmer u.a. (Fn. 81), S. 130.

92 René Pahud de Mortanges, Zwischen religiöser Pluralisierung und Säkularisierung. Aktuelle Entwicklungen bei der staatlichen Anerkennung von Religionsgemeinschaften, in: ders. (Hrsg.), Staatliche Anerkennung von Religionsgemeinschaften. Zukunfts- oder Auslaufmodell?, Zürich/Basel/Genf 2015, S. 11-24, 21 f. 
Anerkennung vielfach offen steht. Diesem Trend würde es entsprechen, auch die staatliche Finanzierung nicht mehr exklusiv an den Anerkennungsstatus zu koppeln.

34 Mit einer Weiterentwicklung dieser Art wären Chancen verbunden. So könnte durch öffentliche Unterstützung bei nicht-anerkannten Religionsgemeinschaften eine Professionalisierung organisatorischer Strukturen erreicht werden. Diese erscheint bei einigen Religionsgemeinschaften unabdingbar, da die allein ehrenamtliche Basis, die heute vielfach besteht, den vielfältigen Anforderungen oft nicht mehr gerecht wird, denen grosse nicht-anerkannte Religionsgemeinschaften gegenüberstehen. Für den Staat eröffnete sich durch eine breiter angelegte Finanzierung zudem die Möglichkeit, stärker Einfluss auf die Entwicklung dieser Gemeinschaften zu nehmen. Eine weiter gefasste öffentliche Finanzierung könnte die Grundlage verbindlicherer Beziehungen bilden, in denen Leistungen und Gegenleistungen klarer definiert wären. 